\title{
ON SECOND- ORDER SYMMETRIC DUALITY FOR A CLASS OF MULTIOBJECTIVE FRACTIONAL PROGRAMMING PROBLEM
}

\author{
DEO BRAT OJHA
}

\begin{abstract}
This article is concerned with a pair of second-order symmetric duals in the context of non-differentiable multiobjective fractional programming problems. We establish the weak and strong duality theorems for the new pair of dual models. Discussion on some special cases shows that results in this paper extend previous work in this area.
\end{abstract}

\section{Introduction}

Symmetric duality in non-linear programming was introduced by Dorn [11], who defined a mathematical programming problem in which the dual of the dual is primal. Subsequently, Dantzig, Eisenberg and Cottle [10] and Mond [19] formulated a pair of symmetric dual programs for scalar function $f(x, y)$ that is convex in the first variable and is concave in the second variable. then Weir and Mond [27] give another different pair of symmetric dual non-linear programs in which a weaker convexity assumptions was imposed on $f$. Chandra, Craven and Mond [6] formulated a pair of symmetric dual fractional programs with certain convexity hypothesis. Weir and Mond discuused symmetric duality in multiobjective programming, Weir presented a pair of symmetric dual multiobjective fractional programming problems and proved symmetric duality theorems under convexity and concavity assumptions. Recently, Yang, Wang and Deng [28] presented symmetric duality for a class of multiobjective fractional programming problem in first order. On the other hand , Mond [19], Bector and Chandra [3], studied second order primal and dual non-linear programs. Later on, Wang [25], generalized the results of Bector and Chandra [3] to non-linear programs involving second order pseudo-convex functions. Then Mond and Schechter [21] constructed two new symmetric dual pairs in which the objectives contain a support function and are therefore non-differentiable .Then Hou and Yang[14] presented second order symmetric duality in non-differentiable programming in which objectives contain a support function and are therefore non-differentiable. In this paper we are motivated to extend the work of Yang, 
Wang and Deng [28] in the light of Hou and Yang [14] multiobjective fractional programming with symmetric duality in second-order. For more details interested reader may consult $[1,2,5,8,9,12,13,15,16,17,18,20,22,24,26]$.

\section{Notations and preliminaries}

The following conventions for vectors in Rn will be used :

$$
\begin{aligned}
& x>y \text { if and only if } x_{i}>y_{i}, \quad i=1,2, \ldots, n ; \\
& x \geqq y \text { if and only if } x_{i} \geqq y_{i}, \quad i=1,2, \ldots, n ; \\
& x \geq y \text { if and only if } x_{i} \geq y_{i}, \quad i=1,2, \ldots, n ;
\end{aligned}
$$

let $f(x, y)$ be a real-valued continuously twice differentiable function defined on an open set in $R^{n} \times R^{m} \rightarrow R$. Let $\nabla_{x} f(x, y)$ and $\nabla_{y} f(x, y)$ denote gradient (column) vectors of $f$ with respect to $x$ and $y$ respectively and $\nabla_{y y} f$ and $\nabla_{y x} f$ denote $(m \times m)$ and $(m \times n)$ matrices of second order partial derivatives respectively.

If $f$ is a twice differentiable function from $R^{n} \times R^{m} \rightarrow R_{k}$, then $\nabla_{x} f(x, y)$ and $\nabla_{y} f(x, y)$ denote respectively the $(n \times k)$ and $(m \times k)$ matrices of first - order partial derivatives.

Definition 2.1. Let $C$ be a compact convex set in $R^{n}$, the support function of $C$ is defined by

$$
S(x / C)=\operatorname{Max} .\left\{x^{T} y, y \in C\right\} .
$$

Definitions 2.2. Let $D$ be a nonempty convex set in $R^{n}$, and let $f: D \rightarrow R$ be convex. Then $z$ is called subgradient of $f$ at $u \in D$ if $f(x) \geq f(u)+z^{T}(x-u)$ for all $x \in D$.

A support function, being convex and everywhere finite has a subdifferential in the sense of convex analysis, that is there exist $z$ such that

$$
S(y / C) \geq S(x / C)+z^{T}(y-x),
$$

for all $x \in C$. The set of all subdifferential $S(x / C)$ is given by

$$
\partial S(x / C)=\left\{z \in C: z^{T} x=S(x / C)\right\} .
$$

For a set $S$, the normal cone to $S$ at a point $x \in S$ is defined by

$$
N_{S}(x)=\left\{y: y^{T}(z-x) \leq 0 \text { for all } z \in S\right\} .
$$

Where $C$ is a compact convex set, $y$ is in $N_{C}(x)$ if and only if $S(y / C)=x^{T} y$ i.e., $x$ is a subdifferential of $S$ at $y$. 
Consider the following multiobjective programming problem Min $f(x)$ subject to $x \in X$ - - - - - - (P) Where $f: R^{n} \rightarrow R^{k}$ and $x \subset R^{n}$.

Definition 2.3. (a) A feasible point $x_{0}$ is said to be an efficient solution of (P) if for any feasible $x$.

$$
f_{i}\left(x_{0}\right) \geq f_{i}(x) \text { for all } i=1,2, \ldots, k \text {, and } x \in X \Rightarrow f_{i}\left(x_{0}\right)=f_{i}(x) \text { for all } i=1,2, \ldots, k .
$$

(b) A feasible point $x$ is said to be properly efficient [14] if it is an efficient solution of (P) and if there exist a scalar $M>0$ such that for all $i, j, f_{i}\left(x_{0}\right)-f_{i}(x) \leq M\left(f_{j}\left(x_{0}\right)-f_{j}(x)\right)$ for some $j$ such that $f_{j}(x)>f_{j}\left(x_{0}\right)$, whenever $x$ is feasible for $(\mathrm{P})$ and $f_{i}(x)<f_{i}\left(x_{0}\right)$.

(c) A feasible point $x_{0}$ is said to be weak efficient solution of (P) [14] if there exist no other feasible point $x$ for which $f\left(x_{0}\right)>f(x)$.

If a feasible point $x_{0}$ is efficient solution then it is clear that it is also a weak efficient solution.

Definition 2.4. Let $\eta: C \times C \rightarrow R(a, b, p, q$ are defined on same as $\eta)$

(a) A differentiable function $\varphi$ defined on set $C \subseteq R^{n}$ is said to be invex in second- order with respect to $\eta$ at $u \in C[14]$ if $\varphi(x)-\varphi(u)+\frac{1}{2} q^{T} \nabla_{u u} \varphi(u) q \geq \eta(x, u)^{T}\left[\nabla_{u} \varphi(u)+\nabla_{u u} \varphi(u) p\right]$ for all $x \in C$.

If $-\varphi$ is invex in second-order with respect to $\eta$ at $u \in C$, then $\varphi$ is said to be incave in second - order with respect to $\eta$ at $u \in C$.

(b) A differentiable function $\varphi$ defined on set $C \subseteq R^{n}$ is said to be pseudo- invex in second order with respect to $\eta$ at $u \in C$ [14] if for all $x \in C$,

$$
\eta(x, u)^{T}\left[\nabla_{u} \varphi(u)+\nabla_{u u} \varphi(u) p\right] \geq 0 \Rightarrow \varphi(x) \geq \varphi(u)-\frac{1}{2} q(x, u)^{T}\left[\nabla_{u u} \varphi(u)\right] q(x, u) .
$$

If $-\varphi$ is pseudo-invex in second-order with respect to $\eta$ at $u \in C$, then $\varphi$ is said to be pseudo incave in second - order with respect to $\eta$ at $u \in C$.

\section{Symmetric duality}

Consider the following pair of symmetric dual problems.

\section{Primal (FP):}

$$
\operatorname{Minimize}\left(\begin{array}{l}
\frac{\left(f_{1}(x, y)+S\left(x / C_{1}\right)-y^{T} z_{1}-\frac{1}{2} a^{T}\left[\nabla_{y y} f_{1}(x, y)\right] a\right)}{\left(g_{1}(x, y)-S\left(x / E_{1}\right)+y^{T} r_{1}+\frac{1}{2} b^{T}\left[\nabla_{y y} g_{1}(x, y)\right] b\right)}, \ldots, \\
\frac{\left(f_{k}(x, y)+S\left(x / C_{k}\right)-y^{T} z_{k}-\frac{1}{2} a^{T}\left[\nabla_{y y} f_{k}(x, y)\right] a\right)}{\left(g_{k}(x, y)-S\left(x / E_{k}\right)+y^{T} r_{k}+\frac{1}{2} b^{T}\left[\nabla_{y y} g_{k}(x, y)\right] b\right)}
\end{array}\right) .
$$


In order to simplify the notation, we introduce

$$
\begin{aligned}
& l=\frac{F_{i}(x, y)}{G_{i}(x, y)}=\left\{\frac{\left(f_{i}(x, y)+S\left(x / C_{i}\right)-y^{T} z_{i}-\frac{1}{2} a^{T}\left[\nabla_{y y} f_{i}(x, y)\right] a\right)}{\left(g_{i}(x, y)-S\left(x / E_{1}\right)+y^{T} r_{i}+\frac{1}{2} b^{T}\left[\nabla_{y y} g_{i}(x, y)\right] b\right)}\right\} \\
& L=\frac{F_{i}(u, v)}{G_{i}(u, v)}=\left\{\frac{\left(f_{i}(u, v)-S\left(\nu / D_{i}\right)+u^{T} w_{i}-\frac{1}{2} p^{T}\left[\nabla_{u u} f_{i}(u, v)\right] p\right)}{\left(g_{i}(u, v)+S\left(v / H_{i}\right)-u^{T} t_{i}+\frac{1}{2} q^{T}\left[\nabla_{u u} g_{i}(u, v)\right] q\right)}\right\}
\end{aligned}
$$

(MFP)

Min. $l=\left(l_{1}, l_{2}, \ldots, l_{k}\right)^{T}$

Subject to

$$
\begin{aligned}
& \left(F_{i}(x, y)-l_{i} G_{i}(x, y)\right)=0 \\
& \sum_{i=1}^{k} \lambda_{i}\left[\nabla_{y}\left(F_{i}(x, y)-l_{i} G_{i}(x, y)\right)-z_{i}+\nabla_{y y}\left(F_{i}(x, y)-l_{i} G_{i}(x, y)\right)\right] \leq 0 \\
& y^{T} \sum_{i=1}^{k} \lambda_{i}\left[\nabla_{y}\left(F_{i}(x, y)-l_{i} G_{i}(x, y)\right)-z_{i}+\nabla_{y y}\left(F_{i}(x, y)-l_{i} G_{i}(x, y)\right)\right] \geq 0 \\
& z_{i} \in D_{i}, r_{i} \in F_{i}, 1 \leq i \leq k \\
& \lambda>0, \lambda^{T} e=1, x \geq 0
\end{aligned}
$$

(MFD)

$$
\text { Maximize } L=\left(L_{1}, L_{2}, \ldots, L_{k}\right)^{T}
$$

Subject to

$$
\begin{aligned}
& \left(F_{i}(u, v)-L_{i} G_{i}(u, v)\right)=0 \\
& \sum_{i=1}^{k} \lambda_{i}\left[\nabla_{u}\left(F_{i}(u, v)-L_{i} G_{i}(u, v)\right)+w_{i}+\nabla_{u u}\left(F_{i}(u, v)-L_{i} G_{i}(u, v)\right)\right] \geq 0 \\
& u^{T} \sum_{i=1}^{k} \lambda_{i}\left[\nabla_{u}\left(F_{i}(u, v)-L_{i} G_{i}(u, v)\right)+w_{i}+\nabla_{u u}\left(F_{i}(u, v)-L_{i} G_{i}(u, v)\right)\right] \leq 0 \\
& w_{i} \in C_{i}, t_{i} \in E_{i}, 1 \leq i \leq k \\
& \lambda>0, \lambda^{T} e=1, v \geq 0
\end{aligned}
$$

Where $e(1,1,1, \ldots, 1)^{T} \in R^{k} ; F_{i}, G_{i}(i=1,2, \ldots, k)$ are thrice differentiable function from $R^{n} \times$ $R^{m}$ to $R, C_{i}, E_{i}(i=1,2, \ldots, k)$ are compact convex sets in $R^{n}$ and $D_{i}, H_{i}(i=1,2, \ldots, k)$ are compact convex sets in $R^{m}$. It is assumed through that in the feasible regions the denominator of the objective function is nonnegative and the numerator is positive.

Now we can prove weak and strong duality theorems for (MFP) and (MFD).

Theorem 3.1. (Weak Duality) 
Let $\left(x, y, \lambda, z_{1}, z_{2}, \ldots, z_{k}, r_{1}, r_{2}, \ldots, r_{k}\right)$ be feasible to (MFP) and let $\left(u, v, \lambda, w_{1}, w_{2}, \ldots, w_{k}\right.$, $t_{1}, t_{2}, \ldots, t_{k}$ ) be feasible to (MFD). Let

$$
\sum_{i=1}^{k} \lambda_{i}\left[f_{i}(\cdot, v)+(\cdot)^{T} w_{i}-L_{i}\left(g_{i}(\cdot, v)-(\cdot)^{T} t_{i}\right)\right]
$$

be second-order pseudoinvex with respect to $\eta_{1}$ at $u$ and

$$
\sum_{i=1}^{k} \lambda_{i}\left[f_{i}(x, \cdot)-(\cdot)^{T} z_{i}-l_{i}\left(g_{i}(x, \cdot)+(\cdot)^{T} r_{i}\right)\right]
$$

be second- order pseudoincave with respect to $\eta_{2}$ at $y$. If

$$
\eta_{1}(x, u)+u \geq 0 \text { and } \eta_{2}(v, y)+y \geq 0 \text { then } l_{i} \nless L_{i} .
$$

Proof. From $\eta_{1}(x, u)+u \geq 0$, and (10) we have

$$
\eta_{1}(x, u)^{T} \sum_{i=1}^{k} \lambda_{i}\left[\nabla_{u}\left(F_{i}(u, v)-L_{i} G_{i}(u, v)\right)+w_{i}+\nabla_{u u}\left(F_{i}(u, v)-L_{i} G_{i}(u, v)\right)\right] \geq 0 .
$$

Second- order pseudoinvexity with respect to $\eta_{1}$ at $u$ of (13), it follows that

$$
\begin{aligned}
& \sum_{i=1}^{k} \lambda_{i}\left[f_{i}(x, v)+(x)^{T} w_{i}-L_{i}\left(g_{i}(x, v)+S\left(v / H_{i}\right)-(x)^{T} t_{i}\right)\right] \\
& \geq \sum_{i=1}^{k} \lambda_{i}\left[f_{i}(u, v)+(u)^{T} w_{i}-\frac{1}{2} p^{T}\left\{\nabla_{u u}\left\{f_{i}(u, v)\right\}\right\} p\right. \\
& \left.\quad-L_{i}\left(g_{i}(u, v)+S\left(v / H_{i}\right)-(u)^{T} t_{i}-\frac{1}{2} q^{T}\left\{\nabla_{u u}\left\{g_{i}(u, v)\right\}\right\} q\right)\right] \\
& \Rightarrow \sum_{i=1}^{k} \lambda_{i}\left[f_{i}(x, v)-S\left(v / D_{i}\right)+(x)^{T} w_{i}-L_{i}\left(g_{i}(x, v)+S\left(v / H_{i}\right)-(x)^{T} t_{i}\right)\right] \geq 0 .
\end{aligned}
$$

Since $x^{T} w_{i} \leq S\left(x / C_{i}\right), w_{i} \in C_{i}(1 \leq i \leq k)$, by (11) and (16), we have

$$
\sum_{i=1}^{k} \lambda_{i}\left[f_{i}(x, v)+S\left(x / C_{i}\right)-S\left(v / D_{i}\right)-L_{i}\left(g_{i}(x, v)+S\left(v / H_{i}\right)-(x)^{T} t_{i}\right)\right] \geq 0 .
$$

From

$x^{T} \leq S\left(x / E_{i}\right), t_{i} \in E_{i}, v^{T} r_{i} \leq S\left(v / H_{i}\right), r_{i} \in H_{i}, f_{i}(u, v)-S\left(v / D_{i}\right)+u^{T} w_{i}-\frac{1}{2} p^{T}\left[\nabla_{u u} f_{i}(u, v)\right] p \geq 0$, and

$$
\left(g_{i}(u, v)+S\left(v / H_{i}\right)-u^{T} t_{i}+\frac{1}{2} q^{T}\left[\nabla_{u u} g_{i}(u, v)\right] q\right) \geq 0,
$$

by (17), we obtain

$$
\sum_{i=1}^{k} \lambda_{i}\left[f_{i}(x, v)+S\left(x / C_{i}\right)-S\left(v / D_{i}\right)-L_{i}\left(g_{i}(x, v)-S\left(x / E_{i}\right)-(v)^{T} r_{i}\right)\right] \geq 0 .
$$


By (3), (4) and $\eta_{2}(\nu, y)+y \geq 0$, we have

$$
\eta_{2}(\nu, y)^{T} \sum_{i=1}^{k} \lambda_{i}\left[\nabla_{y}\left(F_{i}(x, y)-l_{i} G_{i}(x, y)\right)-z_{i}+\nabla_{y y}\left(F_{i}(x, y)-l_{i} G_{i}(x, y)\right)\right] \leq 0 .
$$

Second- order pseudoincavity with respect to $\eta_{2}$ at $y$ of (14), it follows that

$$
\begin{aligned}
& \sum_{i=1}^{k} \lambda_{i}\left[f_{i}(x, v)-(v)^{T} z_{i}-l_{i}\left(g_{i}(x, v)-S\left(x / E_{i}\right)+(v)^{T} r_{i}\right)\right] \\
& \leq \sum_{i=1}^{k} \lambda_{i}\left[f_{i}(x, y)-(y)^{T} z_{i}-\frac{1}{2} a^{T} \nabla_{y y} f_{i}(x, y) a\right. \\
& \left.\quad-l_{i}\left(g_{i}(x, y)-S\left(x / E_{i}\right)-(y)^{T} r_{i}-\frac{1}{2} b^{T} \nabla_{y y} g_{i}(x, y) b\right)\right] \\
& \Rightarrow \sum_{i=1}^{k} \lambda_{i}\left[f_{i}(x, v)+S\left(x / C_{i}\right)-(v)^{T} z_{i}-l_{i}\left(g_{i}(x, v)-S\left(x / E_{i}\right)+(v)^{T} r_{i}\right)\right] \leq 0 .
\end{aligned}
$$

Since $v^{T} z_{i} \leq S\left(v / D_{i}\right), Z_{i} \in D_{i}(1 \leq i \leq k)$ and by (20), we obtain

$$
\sum_{i=1}^{k} \lambda_{i}\left[f_{i}(x, v)+S\left(x / C_{i}\right)-S\left(v / D_{i}\right)-l_{i}\left(g_{i}(x, v)-S\left(x / E_{i}\right)+(v)^{T} r_{i}\right)\right] \leq 0 .
$$

Combining (18) and (21), we have

$$
\sum_{i=1}^{k} \lambda_{i}\left[l_{i}-L_{i}\right]\left(g_{i}(x, v)-S\left(x, E_{i}\right)+(v)^{T} r_{i}\right) \geq 0 .
$$

Since $\lambda>0$ and $\left(g_{i}(x, v)-S\left(x, E_{i}\right)+(v)^{T} r_{i}\right)>0(i=1,2, \ldots, k)$, (22) implies

$$
\begin{aligned}
& \frac{\left(f_{1}(x, y)+S\left(x / C_{1}\right)-y^{T} z_{1}\right)-\frac{1}{2} a^{T}\left[\nabla_{y y} f_{1}(x, y)\right] a}{\left(g_{1}(x, y)-S\left(x / E_{1}\right)+y^{T} r_{1}\right)+\frac{1}{2} b^{T}\left[\nabla_{y y} g_{1}(x, y)\right] b}, \ldots, \\
& \quad \frac{\left(f_{k}(x, y)+S\left(x / C_{k}\right)-y^{T} z_{k}\right)-\frac{1}{2} a^{T}\left[\nabla_{y y} f_{1}(x, y)\right] a}{\left(g_{k}(x, y)-S\left(x / E_{k}\right)+y^{T} r_{k}\right)+\frac{1}{2} b^{T}\left[\nabla_{y y} g_{1}(x, y)\right] b} \\
& > \\
& \quad \frac{\left(f_{1}(u, v)-S\left(v / D_{1}\right)+u^{T} w_{1}\right)-\frac{1}{2} p^{T}\left[\nabla_{u u} f_{i}(u, v)\right] p}{\left(g_{1}(u, v)+S\left(v / H_{1}\right)-u^{T} t_{1}\right)+\frac{1}{2} q^{T}\left[\nabla_{u u} g_{i}(u, v)\right] q}, \ldots, \\
& \left.\quad \frac{\left(f_{k}(u, v)-S\left(v / D_{k}\right)+u^{T} w_{k}\right)-\frac{1}{2} p^{T}\left[\nabla_{u u} f_{i}(u, v)\right] p}{\left(g_{k}(u, v)+S\left(v / H_{k}\right)-u^{T} t_{k}\right)+\frac{1}{2} q^{T}\left[\nabla_{u u} g_{i}(u, v)\right] q}\right) .
\end{aligned}
$$

Theorem.(Strong Duality) Let $\left(x^{\prime}, y^{\prime}, \lambda^{\prime}, z_{1}^{\prime}, z_{2}^{\prime}, \ldots, z_{k}^{\prime}\right)$ be a properly efficient solution to ( $F P$ ) and fix $\lambda=\lambda^{\prime}$ in (FD). Suppose that all the condition in Theorem 3.1 are satisfied, furthermore assume that

(I) $\quad \sum_{i=1}^{k} \lambda_{i}^{\prime}\left\{\nabla_{y y} f_{i}\left(x^{\prime}, y^{\prime}\right)-\left[\left(\frac{\nabla_{y} f_{i}\left(x^{\prime}, y^{\prime}\right)-z_{i}}{g_{i}\left(x^{\prime}, y^{\prime}\right)-S\left(x^{\prime} / E_{i}\right)+y^{T} r_{i}^{\prime}}\right)\right.\right.$ 


$$
\begin{aligned}
& \left.-\left(\frac{\left(\nabla_{y} g_{i}\left(x^{\prime}, y^{\prime}\right)+r_{i}^{\prime}\right)\left(f_{i}\left(x^{\prime}, y^{\prime}\right)+S\left(x^{\prime} / C_{i}\right)-y^{\prime T} z_{i}^{\prime}\right)}{\left(g_{i}\left(x^{\prime}, y^{\prime}\right)-S\left(x^{\prime} / E_{i}\right)+y^{\prime T} r_{i}^{\prime}\right)^{2}}\right)\right]\left(\nabla_{y} g_{i}\left(x^{\prime}, y^{\prime}\right)+r_{i}^{\prime}\right)^{T} \\
& \left.-\left[\frac{f_{i}\left(x^{\prime}, y^{\prime}\right)+S\left(x^{\prime} / C_{i}\right)-y^{\prime T} z_{i}^{\prime}}{g_{i}\left(x^{\prime}, y^{\prime}\right)-S\left(x^{\prime} / E_{i}\right)+y^{\prime} r_{i}^{\prime}}\right]\left(\nabla_{y y} g_{i}\left(x^{\prime}, y^{\prime}\right)\right)\right\}
\end{aligned}
$$

is positive or negative definite.

(II) $\left\{\left(\nabla_{y} f_{i}\left(x^{\prime}, y^{\prime}\right)-z_{i}^{\prime}\right)-\left[\frac{f_{i}\left(x^{\prime}, y^{\prime}\right)+S\left(x^{\prime} / C_{i}\right)-y^{\prime T} z_{i}^{\prime}}{g_{i}\left(x^{\prime}, y^{\prime}\right)-S\left(x^{\prime} / E_{i}\right)+y^{\prime T} r_{i}^{\prime}}\right]\left(\nabla_{y} g_{i}\left(x^{\prime}, y^{\prime}\right)+r_{i}^{\prime}\right): i=1,2, \ldots, k\right\}$

is linearly independent.

Then there exist $w_{i}^{\prime} \in R^{n}, t_{i}^{\prime} \in R^{m}, i=1,2, \ldots, k$ such that $\left(x^{\prime}, y^{\prime}, \lambda^{\prime}, w_{1}^{\prime}, w_{2}^{\prime}, \ldots, w_{k}^{\prime}, t_{1}^{\prime}, t_{2}^{\prime}, \ldots, t_{k}^{\prime}\right)$ is a properly efficient solution of $(F D)$.

Proof. Since $\left(x^{\prime}, y^{\prime}, \lambda^{\prime}, z_{1}^{\prime}, z_{2}^{\prime}, \ldots, z_{k}^{\prime}, r_{1}^{\prime}, r_{2}^{\prime}, \ldots, r_{k}^{\prime}\right)$ be a properly efficient solution to (FP), it is weak minimum of (FP). Hence, there exist $\alpha, \beta, s, \mu \in R^{k}, \eta \in R, w_{i}^{\prime} \in R^{n}$, and $t_{i}^{\prime} \in R^{m}$ ( $i=$ $1,2, \ldots, k)$ such that following Fritz-John optimality condition [23] is satisfied at $\left(x^{\prime}, y^{\prime}, \lambda^{\prime}\right.$, $\left.z_{1}^{\prime}, z_{2}^{\prime}, \ldots, z_{k}^{\prime}\right)$

$$
\begin{aligned}
\sum_{i=1}^{k} \alpha_{i}\left[\nabla_{x} f_{i}\left(x^{\prime}, y^{\prime}\right)+w_{i}^{\prime}-\frac{\left(f_{i}\left(x^{\prime}, y^{\prime}\right)+S\left(x^{\prime} / C_{i}\right)-y^{\prime T} z_{i}^{\prime}\right)}{\left(g_{i}\left(x^{\prime}, y^{\prime}\right)-S\left(x^{\prime} / E_{i}\right)+y^{\prime T} r_{i}^{\prime}\right)}\left(\nabla_{x} g_{i}\left(x^{\prime}, y^{\prime}\right)-t_{i}^{\prime}\right)\right] & \\
+ & \left(\beta-\mu y^{\prime}\right)^{T} \sum_{i=1}^{k} \lambda_{i}^{\prime}\left\{\nabla_{y x} f_{i}\left(x^{\prime}, y^{\prime}\right)-\left[\frac{\nabla_{x} f_{i}\left(x^{\prime}, y^{\prime}\right)-w_{i}^{\prime}}{g_{i}\left(x^{\prime}, y^{\prime}\right)-S\left(x^{\prime} / E_{i}\right)+y^{\prime T} r_{i}^{\prime}}\right.\right. \\
& -\frac{\left(\nabla_{x} g_{i}\left(x^{\prime}, y^{\prime}\right)-t_{i}^{\prime}\right)\left(f_{i}\left(x^{\prime}, y^{\prime}\right)+S\left(x^{\prime} / C_{i}\right)-y^{\prime T} z_{i}^{\prime}\right)}{\left(g_{i}\left(x^{\prime}, y^{\prime}\right)-S\left(x^{\prime} / E_{i}\right)+y^{\prime T} r_{i}^{\prime}\right)^{2}}\left(\nabla_{y} g_{i}\left(x^{\prime}, y^{\prime}\right)+r_{i}^{\prime}\right)^{T} \\
& \left.\left.-\frac{f_{i}\left(x^{\prime}, y^{\prime}\right)+S\left(x^{\prime} / C_{i}\right)-y^{\prime} z_{i}^{\prime}}{g_{i}\left(x^{\prime}, y^{\prime}\right)-S\left(x^{\prime} / E_{i}\right)+y^{\prime} r_{i}^{\prime}} \nabla_{x y} g_{i}\left(x^{\prime}, y^{\prime}\right)=s\right]\right\} \\
+ & \nabla_{x}\left\{\sum _ { i = 1 } ^ { k } \lambda _ { i } ^ { \prime } \left[\nabla_{y y} f_{i}\left(x^{\prime}, y^{\prime}\right)-\left(\frac{\nabla_{y} f_{i}\left(x^{\prime}, y^{\prime}\right)-z_{i}}{g_{i}\left(x^{\prime}, y^{\prime}\right)-S\left(x^{\prime} / E_{i}\right)+y^{T} r_{i}^{\prime}}\right)\right.\right. \\
& \left.-\left(\frac{\left(\nabla_{y} g_{i}\left(x^{\prime}, y^{\prime}\right)+r_{i}^{\prime}\right)\left(f_{i}\left(x^{\prime}, y^{\prime}\right)+S\left(x^{\prime} / C_{i}\right)-y^{\prime T} z_{i}^{\prime}\right)}{\left(g_{i}\left(x^{\prime}, y^{\prime}\right)-S\left(x^{\prime} / E_{i}\right)+y^{\prime T} r_{i}^{\prime}\right)^{2}}\right)\right]\left(\nabla_{y} g_{i}\left(x^{\prime}, y^{\prime}\right)+r_{i}^{\prime}\right)^{T} \\
& \left.-\frac{\left[f_{i}\left(x^{\prime}, y^{\prime}\right)+S\left(x^{\prime} / C_{i}\right)-y^{\prime T} z_{i}^{\prime}\right]}{\left[g_{i}\left(x^{\prime}, y^{\prime}\right)-S\left(x^{\prime} / E_{i}\right)+y^{\prime T} r_{i}^{\prime}\right]}\left(\nabla_{y y} g_{i}\left(x^{\prime}, y^{\prime}\right)\right)(\bar{a}+\bar{b})\right\} \\
{[\beta-} & \left.\frac{1}{2} \alpha(\bar{a}+\bar{b})-\mu \bar{y}\right]=s \\
t_{i}^{\prime} \in & E_{i}, w_{i}^{\prime} \in C_{i}, \forall i \in\{1,2, \ldots, k\} \\
x_{i}^{\prime} T= & S\left(x^{\prime} / E_{i}\right), x^{\prime T} w_{i}^{\prime}=S\left(x^{\prime} / C_{i}\right), \forall i \in\{1,2, \ldots, k\}
\end{aligned}
$$




$$
\begin{aligned}
& \sum_{i=1}^{k}\left(\alpha_{i}-\mu \lambda_{i}^{\prime}\right)\left[\nabla_{y} f_{i}\left(x^{\prime}, y^{\prime}\right)-z_{i}^{\prime}-\frac{\left(f_{i}\left(x^{\prime}, y^{\prime}\right)+S\left(x^{\prime} / C_{i}\right)-y^{\prime T} z_{i}^{\prime}\right)}{\left(g_{i}\left(x^{\prime}, y^{\prime}\right)-S\left(x^{\prime} / E_{i}\right)+y^{\prime T} r_{i}^{\prime}\right)}\left(\nabla_{y} g_{i}\left(x^{\prime}, y^{\prime}\right)+r_{i}^{\prime}\right)\right] \\
& +\left(\beta-\mu y^{\prime}-\mu(\bar{a}+\bar{b})\right)^{T} \sum_{i=1}^{k} \lambda_{i}^{\prime}\left\{\nabla_{y y} f_{i}\left(x^{\prime}, y^{\prime}\right)-\left[\frac{\nabla_{y} f_{i}\left(x^{\prime}, y^{\prime}\right)-z_{i}^{\prime}}{g_{i}\left(x^{\prime}, y^{\prime}\right)-S\left(x^{\prime} / E_{i}\right)+y^{\prime T} r_{i}^{\prime}}\right.\right. \\
& \left.-\frac{\left(\nabla_{y} g_{i}\left(x^{\prime}, y^{\prime}\right)+r_{i}^{\prime}\right)\left(f_{i}\left(x^{\prime}, y^{\prime}\right)+S\left(x^{\prime} / C_{i}\right)-y^{\prime T} z_{i}^{\prime}\right)}{\left(g_{i}\left(x^{\prime}, y^{\prime}\right)-S\left(x^{\prime} / E_{i}\right)+y^{\prime T} r_{i}^{\prime}\right)^{2}}\right]\left(\nabla_{y} g_{i}\left(x^{\prime}, y^{\prime}\right)+r_{i}^{\prime}\right)^{T} \\
& \left.-\frac{\left[f_{i}\left(x^{\prime}, y^{\prime}\right)+S\left(x^{\prime} / C_{i}\right)-y^{\prime T} z_{i}^{\prime}\right]}{\left[g_{i}\left(x^{\prime}, y^{\prime}\right)-S\left(x^{\prime} / E_{i}\right)+y^{\prime T} r_{i}^{\prime}\right]} \nabla_{y y} g_{i}\left(x^{\prime}, y^{\prime}\right)\right\} \\
& +\nabla_{y}\left\{\sum _ { i = 1 } ^ { k } \lambda _ { i } ^ { \prime } \left[\nabla_{y y} f_{i}\left(x^{\prime}, y^{\prime}\right)-\left(\frac{\nabla_{y} f_{i}\left(x^{\prime}, y^{\prime}\right)-z_{i}}{g_{i}\left(x^{\prime}, y^{\prime}\right)-S\left(x^{\prime} / E_{i}\right)+y^{T} r_{i}^{\prime}}\right)\right.\right. \\
& \left.-\left(\frac{\left(\nabla_{y} g_{i}\left(x^{\prime}, y^{\prime}\right)+r_{i}^{\prime}\right)\left(f_{i}\left(x^{\prime}, y^{\prime}\right)+S\left(x^{\prime} / C_{i}\right)-y^{\prime T} z_{i}^{\prime}\right)}{\left(g_{i}\left(x^{\prime}, y^{\prime}\right)-S\left(x^{\prime} / E_{i}\right)+y^{\prime T} r_{i}^{\prime}\right)^{2}}\right)\right]\left(\nabla_{y} g_{i}\left(x^{\prime}, y^{\prime}\right)+r_{i}^{\prime}\right)^{T} \\
& \left.-\frac{\left[f_{i}\left(x^{\prime}, y^{\prime}\right)+S\left(x^{\prime} / C_{i}\right)-y^{\prime T} z_{i}^{\prime}\right]}{\left[g_{i}\left(x^{\prime}, y^{\prime}\right)-S\left(x^{\prime} / E_{i}\right)+y^{\prime T} r_{i}^{\prime}\right]}\left(\nabla_{y y} g_{i}\left(x^{\prime}, y^{\prime}\right)\right)(\bar{a}+\bar{b})\right\} \\
& {\left[\beta-\frac{1}{2} \alpha(\bar{a}+\bar{b})-\mu \bar{y}\right]} \\
& \sum_{i=1}^{k} \lambda_{i}^{\prime}\left\{\nabla_{y y} f_{i}\left(x^{\prime}, y^{\prime}\right)-\left[\left(\frac{\nabla_{y} f_{i}\left(x^{\prime}, y^{\prime}\right)-z_{i}^{\prime}}{g_{i}\left(x^{\prime}, y^{\prime}\right)-S\left(x^{\prime} / E_{i}\right)+y^{T} r_{i}^{\prime}}\right)\right.\right. \\
& \left.-\left(\frac{\left(\nabla_{y} g_{i}\left(x^{\prime}, y^{\prime}\right)+r_{i}^{\prime}\right)\left(f_{i}\left(x^{\prime}, y^{\prime}\right)+S\left(x^{\prime} / C_{i}\right)-y^{\prime T} z_{i}^{\prime}\right)}{\left(g_{i}\left(x^{\prime}, y^{\prime}\right)-S\left(x^{\prime} / E_{i}\right)+y^{\prime} r_{i}^{\prime}\right)^{2}}\right)\right]\left(\nabla_{y} g_{i}\left(x^{\prime}, y^{\prime}\right)+r_{i}^{\prime}\right)^{T} \\
& \left.-\frac{\left[f_{i}\left(x^{\prime}, y^{\prime}\right)+S\left(x^{\prime} / C_{i}\right)-y^{\prime T} z_{i}^{\prime}\right]}{\left[g_{i}\left(x^{\prime}, y^{\prime}\right)-S\left(x^{\prime} / E_{i}\right)+y^{\prime} r_{i}^{\prime}\right]}\left(\nabla_{y y} g_{i}\left(x^{\prime}, y^{\prime}\right)\right)\right\}[\alpha(\bar{a}+\bar{b})-\beta+\mu \bar{y}]=0
\end{aligned}
$$

for all $i \in\{1,2, \ldots, k\}$.

$$
\begin{aligned}
& \alpha_{i} y^{\prime}+\lambda_{i}^{\prime}\left(\beta-\eta y^{\prime}\right)^{T} \in N_{D_{i}}\left(z_{i}\right) \text { for all } i \in\{1,2, \ldots, k\} \\
& \begin{array}{r}
\alpha_{i} y^{\prime} \frac{f_{i}\left(x^{\prime}, y^{\prime}\right)+S\left(x^{\prime} / C_{i}\right)-y^{\prime T} z_{i}^{\prime}}{g_{i}\left(x^{\prime}, y^{\prime}\right)-S\left(x^{\prime} / E_{i}\right)+y^{\prime T} r_{i}^{\prime}}-\lambda_{i}^{\prime}\left(\beta-\eta y^{\prime}\right) \frac{f_{i}\left(x^{\prime}, y^{\prime}\right)+S\left(x^{\prime} / C_{i}\right)-y^{\prime T} z_{i}^{\prime}}{g_{i}\left(x^{\prime}, y^{\prime}\right)-S\left(x^{\prime} / E_{i}\right)+y^{\prime T} r_{i}^{\prime}} \\
\quad \times\left(\left[\nabla_{y} g_{i}\left(x^{\prime}, y^{\prime}\right)+r_{i}^{\prime}\right] y^{\prime}-1\right) \in N_{H_{i}}\left(r_{i}\right), \forall i \in\{1,2, \ldots, k\}
\end{array} \\
& \beta^{T} \sum_{i=1}^{k} \lambda_{i}^{\prime}\left\{\nabla_{y} f_{i}\left(x^{\prime}, y^{\prime}\right)-z_{i}^{\prime}-\frac{f_{i}\left(x^{\prime}, y^{\prime}\right)+S\left(x^{\prime} / C_{i}\right)-y^{\prime T} z_{i}^{\prime}}{g_{i}\left(x^{\prime}, y^{\prime}\right)-S\left(x^{\prime} / E_{i}\right)+y^{\prime T} r_{i}^{\prime}}\left\{\nabla_{y} g_{i}\left(x^{\prime}, y^{\prime}\right)+r_{i}^{\prime}\right\}\right\} \\
& +\sum_{i=1}^{k} \lambda_{i}^{\prime}\left\{\nabla_{y y} f_{i}\left(x^{\prime}, y^{\prime}\right)-\left[\frac{\nabla_{y} f_{i}\left(x^{\prime}, y^{\prime}\right)-z_{i}}{g_{i}\left(x^{\prime}, y^{\prime}\right)-S\left(x^{\prime} / E_{i}\right)+y^{T} r_{i}^{\prime}}\right]\right. \\
& -\left[\frac{\left(\nabla_{y} g_{i}\left(x^{\prime}, y^{\prime}\right)+r_{i}^{\prime}\right)\left(f_{i}\left(x^{\prime}, y^{\prime}\right)+S\left(x^{\prime} / C_{i}\right)-y^{\prime T} z_{i}^{\prime}\right)}{\left(g_{i}\left(x^{\prime}, y^{\prime}\right)-S\left(x^{\prime} / E_{i}\right)+y^{\prime T} r_{i}^{\prime}\right)^{2}}\right]\left(\nabla_{y} g_{i}\left(x^{\prime}, y^{\prime}\right)+r_{i}^{\prime}\right)^{T}
\end{aligned}
$$




$$
\begin{aligned}
& \left.-\frac{\left[f_{i}\left(x^{\prime}, y^{\prime}\right)+S\left(x^{\prime} / C_{i}\right)-y^{\prime T} z_{i}^{\prime}\right]}{\left[g_{i}\left(x^{\prime}, y^{\prime}\right)-S\left(x^{\prime} / E_{i}\right)+y^{\prime T} r_{i}^{\prime}\right]}\left(\nabla_{y y} g_{i}\left(x^{\prime}, y^{\prime}\right)\right)(\bar{a}+\bar{b})\right\}=0 \\
& \eta y^{\prime T} \sum_{i=1}^{k} \lambda_{i}^{\prime}\left\{\nabla_{y} f_{i}\left(x^{\prime}, y^{\prime}\right)-z_{i}^{\prime}-\frac{f_{i}\left(x^{\prime}, y^{\prime}\right)+S\left(x^{\prime} / C_{i}\right)-y^{\prime T} z_{i}^{\prime}}{g_{i}\left(x^{\prime}, y^{\prime}\right)-S\left(x^{\prime} / E_{i}\right)+y^{\prime T} r_{i}^{\prime}}\left[\nabla_{y} g_{i}\left(x^{\prime}, y^{\prime}\right)+r_{i}^{\prime}\right]\right. \\
& +\left[\nabla_{y y} f_{i}\left(x^{\prime}, y^{\prime}\right)-\left[\left(\frac{\nabla_{y} f_{i}\left(x^{\prime}, y^{\prime}\right)-z_{i}}{g_{i}\left(x^{\prime}, y^{\prime}\right)-S\left(x^{\prime} / E_{i}\right)+y^{T} r_{i}^{\prime}}\right)\right.\right. \\
& \left.\quad-\left(\frac{\left(\nabla_{y} g_{i}\left(x^{\prime}, y^{\prime}\right)+r_{i}^{\prime}\right)\left(f_{i}\left(x^{\prime}, y^{\prime}\right)+S\left(x^{\prime} / C_{i}\right)-y^{\prime T} z_{i}^{\prime}\right)}{\left(g_{i}\left(x^{\prime}, y^{\prime}\right)-S\left(x^{\prime} / E_{i}\right)+y^{\prime T} r_{i}^{\prime}\right)^{2}}\right)\right]\left(\nabla_{y} g_{i}\left(x^{\prime}, y^{\prime}\right)+r_{i}^{\prime}\right)^{T} \\
& \left.\left.\quad-\frac{\left[f_{i}\left(x^{\prime}, y^{\prime}\right)+S\left(x^{\prime} / C_{i}\right)-y^{\prime T} z_{i}^{\prime}\right]}{\left[g_{i}\left(x^{\prime}, y^{\prime}\right)-S\left(x^{\prime} / E_{i}\right)+y^{\prime T} r_{i}^{\prime}\right]}\left(\nabla_{y y} g_{i}\left(x^{\prime}, y^{\prime}\right)\right)(\bar{a}+\bar{b})\right]\right\}=0 \\
& s^{T} x^{\prime}=0 \\
& \gamma^{T} \lambda^{\prime}=0 \\
& (\alpha, \beta, s, \mu, \gamma) \geq 0 \\
& (\alpha, \beta, s, \mu, \gamma) \neq 0 .
\end{aligned}
$$

Since $\lambda^{\prime}>0$ and $\gamma \geq 0$, (34) implies $\gamma=0$.

Consequently, (28) yields.

Multiplying (27) by $[\alpha(\bar{a}+\bar{b})-\beta+\mu \bar{y}]$ from left and by (37), we have

$$
\begin{gathered}
\{\alpha(\bar{a}+\bar{b})-\beta+\mu \bar{y})\}^{T} \sum_{i=1}^{k} \lambda_{i}^{\prime}\left\{\nabla_{y y} f_{i}\left(x^{\prime}, y^{\prime}\right)-\left[\frac{\nabla_{y} f_{i}\left(x^{\prime}, y^{\prime}\right)-z_{i}^{\prime}}{g_{i}\left(x^{\prime}, y^{\prime}\right)-S\left(x^{\prime} / E_{i}\right)+y^{\prime T} r_{i}^{\prime}}\right.\right. \\
\left.-\frac{\left(\nabla_{y} g_{i}\left(x^{\prime}, y^{\prime}\right)+r_{i}^{\prime}\right)\left(f_{i}\left(x^{\prime}, y^{\prime}\right)+S\left(x^{\prime} / C_{i}\right)-y^{\prime T} z_{i}^{\prime}\right)}{\left(g_{i}\left(x^{\prime}, y^{\prime}\right)-S\left(x^{\prime} / E_{i}\right)+y^{\prime T} r_{i}^{\prime}\right)^{2}}\right]\left(\nabla_{y} g_{i}\left(x^{\prime}, y^{\prime}\right)+r_{i}^{\prime}\right)^{T} \\
\left.-\frac{\left(f_{i}\left(x^{\prime}, y^{\prime}\right)+S\left(x^{\prime} / C_{i}\right)-y^{\prime T} z_{i}^{\prime}\right)}{\left(g_{i}\left(x^{\prime}, y^{\prime}\right)-S\left(x^{\prime} / E_{i}\right)+y^{\prime T} r_{i}^{\prime}\right)} \nabla_{y y} g_{i}\left(x^{\prime}, y^{\prime}\right)\right\}\{\alpha(\bar{a}+\bar{b})-\beta+\mu \bar{y}\}=0
\end{gathered}
$$

By assumption (I), yields that $\beta=\{\alpha(\bar{a}+\bar{b})+\mu \bar{y}\}$.

From (27) and (38), we have

$$
\begin{aligned}
& \sum_{i=1}^{k}\left(\alpha_{i}-\eta \lambda_{i}^{\prime}\right)\left[\nabla_{y} f_{i}\left(x^{\prime}, y^{\prime}\right)-z_{i}^{\prime}-\frac{f_{i}\left(x^{\prime}, y^{\prime}\right)+S\left(x^{\prime} / C_{i}\right)-y^{\prime T} z_{i}^{\prime}}{g_{i}\left(x^{\prime}, y^{\prime}\right)-S\left(x^{\prime} / E_{i}\right)+y^{\prime T} r_{i}^{\prime}}\left(\nabla_{y} g_{i}\left(x^{\prime}, y^{\prime}\right)+r_{i}^{\prime}\right)\right] \\
& +\sum_{i=1}^{k} \lambda_{i}^{\prime}\left\{\nabla_{y y} f_{i}\left(x^{\prime}, y^{\prime}\right)-\left[\left(\frac{\nabla_{y} f_{i}\left(x^{\prime}, y^{\prime}\right)-z_{i}}{g_{i}\left(x^{\prime}, y^{\prime}\right)-S\left(x^{\prime} / E_{i}\right)+y^{T} r_{i}^{\prime}}\right)\right.\right. \\
& \left.-\left(\frac{\left(\nabla_{y} g_{i}\left(x^{\prime}, y^{\prime}\right)+r_{i}^{\prime}\right)\left(f_{i}\left(x^{\prime}, y^{\prime}\right)+S\left(x^{\prime} / C_{i}\right)-y^{\prime T} z_{i}^{\prime}\right)}{\left(g_{i}\left(x^{\prime}, y^{\prime}\right)-S\left(x^{\prime} / E_{i}\right)+y^{\prime T} r_{i}^{\prime}\right)^{2}}\right)\right]\left(\nabla_{y} g_{i}\left(x^{\prime}, y^{\prime}\right)+r_{i}^{\prime}\right)^{T} \\
& \left.-\frac{\left[f_{i}\left(x^{\prime}, y^{\prime}\right)+S\left(x^{\prime} / C_{i}\right)-y^{\prime T} z_{i}^{\prime}\right]}{\left[g_{i}\left(x^{\prime}, y^{\prime}\right)-S\left(x^{\prime} / E_{i}\right)+y^{\prime T} r_{i}^{\prime}\right]}\left(\nabla_{y y} g_{i}\left(x^{\prime}, y^{\prime}\right)\right)\right\}[\alpha(\bar{a}+\bar{b})-\beta+\mu \bar{y}]=0
\end{aligned}
$$


According to assumption (II), (39) implies $\alpha_{i}=\mu \lambda_{i}^{\prime}, i=1,2, \ldots, k$.

If $\mu=0$, then $\alpha_{i}=0(i=1,2, \ldots, k)$. From (37), $\beta=0$. From (23), $s=0$. From (27), $\gamma_{i}=0$ $(i=1,2, \ldots, k)$. Thus $(\alpha, \beta, s, \mu, \gamma)=0$ contradicting condition (36). Hence, $\mu>0$. From (40) and $\lambda^{\prime}>0$, we have that $\alpha_{i}>0(i=1,2, \ldots, k)$. By (24), (38)and (40), we obtain

$$
\begin{aligned}
\sum_{i=1}^{k} & \lambda_{i}\left[\nabla_{x} f_{i}\left(x^{\prime}, y^{\prime}\right)+w_{i}^{\prime}-\frac{f_{i}\left(x^{\prime}, y^{\prime}\right)+S\left(x^{\prime} / C_{i}\right)-y^{\prime T} z_{i}^{\prime}}{g_{i}\left(x^{\prime}, y^{\prime}\right)-S\left(x^{\prime} / E_{i}\right)+y^{\prime T} r_{i}^{\prime}}\left(\nabla_{x} g_{i}\left(x^{\prime}, y^{\prime}\right)-t_{i}^{\prime}\right)\right] \\
+ & \sum_{i=1}^{k} \lambda_{i}^{\prime}\left\{\nabla_{y y} f_{i}\left(x^{\prime}, y^{\prime}\right)-\left[\left(\frac{\nabla_{y} f_{i}\left(x^{\prime}, y^{\prime}\right)-z_{i}}{g_{i}\left(x^{\prime}, y^{\prime}\right)-S\left(x^{\prime} / E_{i}\right)+y^{T} r_{i}^{\prime}}\right)\right.\right. \\
& \left.-\left(\frac{\left(\nabla_{y} g_{i}\left(x^{\prime}, y^{\prime}\right)+r_{i}^{\prime}\right)\left(f_{i}\left(x^{\prime}, y^{\prime}\right)+S\left(x^{\prime} / C_{i}\right)-y^{\prime} z_{i}^{\prime}\right)}{\left(g_{i}\left(x^{\prime}, y^{\prime}\right)-S\left(x^{\prime} / E_{i}\right)+y^{\prime T} r_{i}^{\prime}\right)^{2}}\right)\right]\left(\nabla_{y} g_{i}\left(x^{\prime}, y^{\prime}\right)+r_{i}^{\prime}\right)^{T} \\
& \left.-\frac{\left[f_{i}\left(x^{\prime}, y^{\prime}\right)+S\left(x^{\prime} / C_{i}\right)-y^{\prime} z_{i}^{\prime}\right]}{\left[g_{i}\left(x^{\prime}, y^{\prime}\right)-S\left(x^{\prime} / E_{i}\right)+y^{\prime T} r_{i}^{\prime}\right]}\left(\nabla_{y y} g_{i}\left(x^{\prime}, y^{\prime}\right)\right)\right\}[\alpha(\bar{a}+\bar{b})-\beta+\mu \bar{y}]=s / \mu \geq 0 .
\end{aligned}
$$

From (41) and (33), it follows that

$$
\begin{aligned}
x^{\prime T} & \sum_{i=1}^{k} \lambda_{i}\left[\nabla_{x} f_{i}\left(x^{\prime}, y^{\prime}\right)+w_{i}^{\prime}-\frac{f_{i}\left(x^{\prime}, y^{\prime}\right)+S\left(x^{\prime} / C_{i}\right)-y^{\prime T} z_{i}^{\prime}}{g_{i}\left(x^{\prime}, y^{\prime}\right)-S\left(x^{\prime} / E_{i}\right)+y^{\prime T} r_{i}^{\prime}}\left(\nabla_{x} g_{i}\left(x^{\prime}, y^{\prime}\right)-t_{i}^{\prime}\right)\right] \\
& +\sum_{i=1}^{k} \lambda_{i}^{\prime}\left\{\nabla_{y y} f_{i}\left(x^{\prime}, y^{\prime}\right)-\left[\left(\frac{\nabla_{y} f_{i}\left(x^{\prime}, y^{\prime}\right)-z_{i}}{g_{i}\left(x^{\prime}, y^{\prime}\right)-S\left(x^{\prime} / E_{i}\right)+y^{T} r_{i}^{\prime}}\right)\right.\right. \\
& \left.-\left(\frac{\left(\nabla_{y} g_{i}\left(x^{\prime}, y^{\prime}\right)+r_{i}^{\prime}\right)\left(f_{i}\left(x^{\prime}, y^{\prime}\right)+S\left(x^{\prime} / C_{i}\right)-y^{\prime T} z_{i}^{\prime}\right)}{\left(g_{i}\left(x^{\prime}, y^{\prime}\right)-S\left(x^{\prime} / E_{i}\right)+y^{\prime T} r_{i}^{\prime}\right)^{2}}\right)\right]\left(\nabla_{y} g_{i}\left(x^{\prime}, y^{\prime}\right)+r_{i}^{\prime}\right)^{T} \\
& \left.-\frac{\left[f_{i}\left(x^{\prime}, y^{\prime}\right)+S\left(x^{\prime} / C_{i}\right)-y^{\prime} z_{i}^{\prime}\right]}{\left[g_{i}\left(x^{\prime}, y^{\prime}\right)-S\left(x^{\prime} / E_{i}\right)+y^{\prime T} r_{i}^{\prime}\right]}\left(\nabla_{y y} g_{i}\left(x^{\prime}, y^{\prime}\right)\right)\right\}[\alpha(\bar{a}+\bar{b})-\beta+\mu \bar{y}]=0 .
\end{aligned}
$$

By (38), (35) and $\mu>0$, we have that $y^{\prime}=\beta / \mu \geq 0$.

From (25), (41), (42) and (43), $\left(x^{\prime}, y^{\prime}, \lambda^{\prime}, w_{1}^{\prime}, w_{2}^{\prime}, \ldots, w_{k}^{\prime}, t_{1}^{\prime}, t_{2}^{\prime}, \ldots, t_{k}^{\prime}\right)$ is feasible for (FD). From (29), (30) and (38), we obtain

$$
y^{\prime T} z_{i}^{\prime}=S\left(x^{\prime} / D_{i}\right), \quad y^{\prime T} r_{i}^{\prime}=S\left(y^{\prime} / H_{i}\right), \quad i=1,2, \ldots, k .
$$

By (26) and (44), we get

$$
\frac{f_{i}\left(x^{\prime}, y^{\prime}\right)+S\left(x^{\prime} / C_{i}\right)-y^{\prime T} z_{i}^{\prime}}{g_{i}\left(x^{\prime}, y^{\prime}\right)-S\left(x^{\prime} / E_{i}\right)+y^{\prime T} r_{i}^{\prime}}=\frac{f_{i}\left(x^{\prime}, y^{\prime}\right)-S\left(y^{\prime} / D_{i}\right)+x^{\prime T} w_{i}^{\prime}}{g_{i}\left(x^{\prime}, y^{\prime}\right)+S\left(y^{\prime} / H_{i}\right)-x^{\prime T} t_{i}^{\prime}} .
$$

Thus, $\left(x^{\prime}, y^{\prime}, \lambda^{\prime}, w_{1}^{\prime}, w_{2}^{\prime}, \ldots, w_{k}^{\prime}, t_{1}^{\prime}, t_{2}^{\prime}, \ldots, t_{k}^{\prime}\right)$ is feasible to (FD) and the objective values of (FP) and (FD) are equal. 
We claim that $\left(x^{\prime}, y^{\prime}, \lambda^{\prime}, w_{1}^{\prime}, w_{2}^{\prime}, \ldots, w_{k}^{\prime}, t_{1}^{\prime}, t_{2}^{\prime}, \ldots, t_{k}^{\prime}\right)$ an efficient solution of (FD). If this would not be the case, there would exist $\left(u, v, w_{1}, w_{2}, \ldots, w_{k}, t_{1}, t_{2}, \ldots, t_{k}\right)$ which would be feasible for (FD)and would satisfy

$$
\frac{f_{i}(u, v)-S\left(v / D_{i}\right)+u^{T} w_{i}}{g_{i}(u, v)+S\left(u / E_{i}\right)-u^{T} t_{i}} \geq \frac{f_{i}\left(x^{\prime}, y^{\prime}\right)-S\left(y^{\prime} / D_{i}\right)+x^{\prime T} w_{i}^{\prime}}{g_{i}\left(x^{\prime}, y^{\prime}\right)+S\left(y^{\prime} / H_{i}\right)-x^{\prime T} t_{i}^{\prime}} \quad \forall i \in\{1,2, \ldots, k\}
$$

and

$$
\frac{f_{j i}(u, v)-S\left(v / D_{j i}\right)+u^{T} w_{j i}}{g_{j}(u, v)+S\left(u / E_{j}\right)-u^{T} t_{j}}>\frac{f_{j i}\left(x^{\prime}, y^{\prime}\right)-S\left(y^{\prime} / D_{j i}\right)+x^{\prime T} w_{j i}^{\prime}}{g_{j}\left(x^{\prime}, y^{\prime}\right)+S\left(y^{\prime} / H_{j}\right)-x^{\prime T} t_{j}^{\prime}} \text { for - some } j \in\{1,2, \ldots, k\} .
$$

From inequalities (26) and (44), we get a contradiction to Theorem 3.1. If $\left(x^{\prime}, y^{\prime}, \lambda^{\prime}, w_{1}^{\prime}, w_{2}^{\prime}, \ldots\right.$, $\left.w_{k}^{\prime}, t_{1}^{\prime}, t_{2}^{\prime}, \ldots, t_{k}^{\prime}\right)$ was not properly efficient, for each fixed real number $M>0$, there would exist a feasible solution $\left(u, v,, w_{1}, w_{2}, \ldots, w_{k}, t_{1}, t_{2}, \ldots, t_{k}\right)$ in (FD) and an index $i$ such that

$$
\begin{aligned}
& \frac{f_{i}(u, v)-S\left(v / D_{i}\right)+u^{T} w_{i}}{g_{i}(u, v)+S\left(u / E_{i}\right)-u^{T} t_{i}}-\frac{f_{i}\left(x^{\prime}, y^{\prime}\right)-S\left(y^{\prime} / D_{i}\right)+x^{\prime T} w_{i}^{\prime}}{g_{i}\left(x^{\prime}, y^{\prime}\right)+S\left(y^{\prime} / H_{i}\right)-x^{\prime T} t_{i}^{\prime}} \\
& \quad>M\left[\frac{f_{j}\left(x^{\prime}, y^{\prime}\right)-S\left(y^{\prime} / D_{j}\right)+x^{\prime T} w_{j}^{\prime}}{g_{j}\left(x^{\prime}, y^{\prime}\right)+S\left(y^{\prime} / H_{j}\right)-x^{\prime T} t_{j}^{\prime}}-\frac{f_{j}(u, v)-S\left(v / D_{j}\right)+u^{T} w_{j}}{g_{j}(u, v)+S\left(u / E_{j}\right)-u^{T} t_{j}}\right]
\end{aligned}
$$

for some $j$ satisfying

$$
\frac{f_{j}\left(x^{\prime}, y^{\prime}\right)-S\left(y^{\prime} / D_{j}\right)+x^{\prime T} w_{j}^{\prime}}{g_{j}\left(x^{\prime}, y^{\prime}\right)+S\left(y^{\prime} / H_{j}\right)-x^{\prime T} t_{j}^{\prime}}>\frac{f_{j}(u, v)-S\left(v / D_{j}\right)+u^{T} w_{j}}{g_{j}(u, v)+S\left(u / E_{j}\right)-u^{T} t_{j}}
$$

whenever

$$
\frac{f_{i}(u, v)-S\left(v / D_{i}\right)+u^{T} w_{i}}{g_{i}(u, v)+S\left(u / E_{i}\right)-u^{T} t_{j}}>\frac{f_{i}\left(x^{\prime}, y^{\prime}\right)-S\left(y^{\prime} / D_{i}\right)+x^{\prime T} w_{i}^{\prime}}{g_{i}\left(x^{\prime}, y^{\prime}\right)+S\left(y^{\prime} / H_{i}\right)-x^{\prime T} t_{i}^{\prime}} x^{T} w_{i}^{\prime}=S\left(x^{\prime} / C_{i}\right)
$$

and $y^{\prime T} z_{i}^{\prime}=S\left(y^{\prime} / D_{i}\right), i=1,2, \ldots, k$. This imply that

$$
\frac{f_{i}(u, v)-S\left(v / D_{i}\right)+u^{T} w_{i}}{g_{i}(u, v)+S\left(u / E_{i}\right)-u^{T} t_{j}}-\frac{f_{i}\left(x^{\prime}, y^{\prime}\right)-S\left(y^{\prime} / D_{i}\right)+x^{\prime T} w_{i}^{\prime}}{g_{i}\left(x^{\prime}, y^{\prime}\right)+S\left(y^{\prime} / H_{i}\right)-x^{\prime T} t_{i}^{\prime}}
$$

can be made arbitrarily large and hence for $\lambda^{\prime}$ with $\lambda_{i}^{\prime}>0$, we have

$$
\sum_{i=1}^{k} \lambda_{i}^{\prime} \frac{f_{i}(u, v)-S\left(v / D_{i}\right)+u^{T} w_{i}}{g_{i}(u, v)+S\left(u / E_{i}\right)-u^{T} t_{j}}>\sum_{i=1}^{k} \lambda_{i}^{\prime} \frac{f_{i}\left(x^{\prime}, y^{\prime}\right)-S\left(y^{\prime} / D_{i}\right)+x^{\prime T} w_{i}^{\prime}}{g_{i}\left(x^{\prime}, y^{\prime}\right)+S\left(y^{\prime} / H_{i}\right)-x^{\prime T} t_{i}^{\prime}}
$$

which contradicts the weak duality Theorem 3.1. This completes the proof.

With a similar technique, we can prove the following theorem.

Theorem 3.4. (Strong Duality) Let $\left(x^{\prime}, y^{\prime}, \lambda^{\prime}, z_{1}^{\prime}, z_{2}^{\prime}, \ldots, z_{k}^{\prime}, r_{1}^{\prime}, r_{2}^{\prime}, \ldots, r_{k}^{\prime}\right)$ be a properly efficient solution to (FP) and fix $\lambda=\lambda^{\prime}$ in (FD). Suppose that all the conditions in Theorem 3.2 are fulfilled. Assume that (I) and (II) of Theorem 3.3 are satisfied then there exist $w_{i}^{\prime} \in R^{n}, t_{i}^{\prime} \in R^{m}$, 
$i=1,2, \ldots, k$, such that $\left(x^{\prime}, y^{\prime}, \lambda^{\prime}, w_{1}^{\prime}, w_{2}^{\prime}, \ldots, w_{k}^{\prime}, t_{1}^{\prime}, t_{2}^{\prime}, \ldots, t_{k}^{\prime}\right)$ is properly efficient solution of (FD).

Remark. In this paper, the same parameter $\lambda \in R^{k}$ appeared in both primal and dual. For a pair of multiobjective Programming problems with $\lambda$ as variable in both programs, some symmetric duality results were given by Chandra and Prasad [7] and some comments were presented by Chandra and Abha[4].

\section{Some Special Cases:}

A frequently occurring example of a nondifferentiable support function is $\left(x^{T} A x\right)^{1 / 2}$, where $A$ is positive semidefinite matrix. It can be easily verified that $\left(x^{T} A x\right)^{1 / 2}=S(x / C)$, where $C=\left\{A y:\left(y^{T} A y\right) \leq 1\right\}$ and that set $C$ is compact convex.

(i) If in the feasible regions $k \equiv 1, g_{i} \equiv 1,\left(x_{i}{ }^{T} B x\right)^{1 / 2}=S\left(x / C_{i}\right)$, where $C_{i}=\left\{B_{i} y:\left(y^{T} B_{i} y\right) \leq\right.$ $1\},\left(x^{T} C x\right)^{1 / 2}=S\left(x / D_{i}\right)$, where $D_{i}=\left\{C_{i} y:\left(y^{T} C_{i} y\right) \leq 1\right\}, i=1,2, \ldots, k$, then programs (FP) and (FD) become a pair of symmetric dual nondifferentiable programs considered by Chandra, Craven, and Mond [6].

(ii) If in (FP) and (FD), $B_{i}=\{0\}$ and $C_{i}=\{0\}, i=1,2, \ldots, k$, and in the feasible regions $g_{i} \equiv$ $1, i=1,2, \ldots, k$, we obtain the symmetric dual multiobjective programming problems studied by Weir and Mond [18].

(iii) If in (FP) and (FD), $k \equiv 1, g_{i} \equiv 1, C_{i}=\{0\}$ and $D_{i}=\{0\}, i=1,2, \ldots, k$, we obtain symmetric dual problems studied by Chandra,Craven, and Mond [6].

(iv) If in (FP) and (FD), $k \equiv 1, g_{i} \equiv 1$, we obtain symmetric dual problems studied by Mond and Schechter [21].

\section{References}

[1] B. Agezzaf, Second-order necessary and sufficient conditions of Kuhn-Tucker type in multiobjective optimization problems, in Twelfth International Conference On Multiobjective Criteria Decision Making, Fernuniversitat Hangen, Hangen Germany, 1995.

[2] B. Agezzaf and M. Hachimi, Second order optimality conditions in multiobjective optimization problems, J. Optim. Theory Appl., 102, (1999), 37-50.

[3] C. R. Bector and S. Chandra, Generalized bonvexity and higher order duality for fractional programming, Opsearch, 24 (1987), 143-154.

[4] S. Chandra and G. Abha, Symmetric duality in multiobjective programs : some remarks on recent results, Eur. J. Oper. Res., 124 (2000), 651-654.

[5] S. Chandra, B. D. Craven and B. Mond, Symmetric dual fractional programming, Z. Oper. Res., 29 (1985), 59-64.

[6] S. Chandra, B. D. Craven and B. Mond, Generalized concavity and duality with a square root term, Optimization, 16 (1985), 654-662.

[7] S. Chandra and M. V. D. Prasad, Symmetric duality in multiobjective programming, J. Austral. Math. Soc. Ser. B, 35(1993), 198-206. 
[8] R. W. Cottle, Symmetric dual quadratic programs, Quart. Appl. Math., 21(1963), 237-243.

[9] B. D. Craven, Lagrangian conditions and quasiduality, Bull. Austral. Math. Soc., 16 (1977), 325-339.

[10] G. G. Dantzig, E. Eisenberg and R. W. Cottle, Symmetric dual nonlinear programs, Pacific J. Math., 15 (1965), 809-812.

[11] W. S. Dorn, A symmetric dual theorem for quadratic programs, J. Oper. Res. Soc. Japan, 2 (1960), 93-97.

[12] T. R. Gulati and I. Ahmad, Second order symmetric duality for nonlinear mixed integer programs, European J. Oper. Res., 101(1997), 122-129.

[13] A. M. Geoffrion, Proper efficiency and the theory of vector optimization, J. Math. Anal. Appl., 22 (1968), 613630.

[14] S. H. Hou and X. M. Yang, On second -order symmetric duality in nondifferentiable programming, J. Math. Anal. Appl., 255 (2001), 491-498.

[15] I. Hussain and G. Abha, Nondifferentiable Symmetric Duality in Fractional Programming, Opsearch, 2000.

[16] M. A. Hanson, On sufficiency of the Kuhn - Tucker conditions, J. Math. Anal. Appl., 80 (1981), 545-550.

[17] R. N. Kaul and S. Kaur, Optimality criteria in nonlinear programming involving nonconvex functions, J.Math. Anal. Appl., 105 (1985), 104-112.

[18] O. L. Mangasarian, Second and higher order duality in nonlinear programming, J. Math. Anal. Appl., 5 (1975), 607-620.

[19] B. Mond, A symmetric dual theory for nonlinear programs, Quart. Appl. Math., 23 (1965), 265-269.

[20] B. Mond and I. Hussain and M. V. D. Prasad, Duality for a class of nondifferentiable multi- objective programs, Util. Math., 39 (1991), 3-19.

[21] B. Mond and M. Schechter, Nondifferentiable symmetric duality, Bull. Austral. Math. Soc., 53 (1996), 177-188.

[22] B. Mond, S. Chandra and M. V. D. Prasad, Symmetric dual nondifferentiable fractional programs, Indian J. Manag. Syst., 13 (1987), 1-10.

[23] M. Schechter, More on subgradient duality, J. Math. Anal. Appl., 71 (1979), 251-262.

[24] I. M. -Minasian Stancu, Fractional programming: Theory, Methods and Applications, in : Mathematics and its Applications, Vol.409, Kluwer Academic, Dordrecht, 1997.

[25] S. Wang, Second order necessary and sufficient conditions in multiobjective programming, Numeri. Funct. Anal. Optim., 12 (1991), 237-252.

[26] T. Wier, Symmetric dual multiobjective fractional programming, J. Austral. Math. Soc. Ser. A, 50 (1991), 67-74.

[27] T. Weir and B. Mond, Symmetric and self duality in multiobjective programming, Asia Pacific J. Oper. Res., 5(1988), 124-133.

[28] X. M. Yang, S. Y. Wang and X. T. Deng, Symmetric duality for a class of multiobjective fractional programming problems, J. Math. Anal. Appl., 274 (2002), 279-295.

Department of Mathematics, Mewar University, 201012, India.

E-mail: ojhdb@yahoo.co.in 